\title{
BMJ Open Exercise training modalities for heart transplant recipients: a systematic review and network meta- analysis protocol
}

\author{
Juliana Beust de Lima (D) , 1,2,3 Douglas dos Santos Soares (D) , 1,3 \\ Filipe Ferrari (D) , ${ }^{1,2,3}$ Nelson Carvas Junior (D) , ${ }^{4}$ Gabriel Carvalho (D) , \\ Santiago Alonso Tobar Leitão (1) ,1,3 Lívia Adams Goldraich (1) ,3,5 \\ Nadine Clausell (D) , ${ }^{1,3,5,6}$ Ricardo Stein (D) 1,2,3,6
}

To cite: de Lima JB, Soares DdS, Ferrari F, et al. Exercise training modalities for heart transplant recipients: a systematic review and network metaanalysis protocol. BMJ Open 2020;10:e044975. doi:10.1136/ bmjopen-2020-044975

- Prepublication history and additional material for this paper are available online. To view these files, please visit the journal online (http://dx.doi. org/10.1136/bmjopen-2020044975).

Received 22 September 2020 Revised 01 December 2020 Accepted 02 December 2020

Check for updates

(C) Author(s) (or their employer(s)) 2020. Re-use permitted under CC BY-NC. No commercial re-use. See rights and permissions. Published by BMJ.

For numbered affiliations see end of article.

Correspondence to

MSc Juliana Beust de Lima;

julianabeustdelima@gmail.com

\section{ABSTRACT}

Introduction Heart transplantation is the gold standard treatment for selected patients with end-stage heart failure. Although this procedure can improve quality and prolong life expectancy, several of these patients persist with decreased exercise tolerance. Evidence suggests that exercise training can bring multifactorial benefits to heart transplant (HTx) recipients. However, it is unclear that exercise modality should be preferred. Therefore, the aim of this systematic review and network meta-analysis is to compare the efficacy and safety of different training modalities in HTx recipients.

Methods and analysis We will perform a comprehensive literature search in PubMed/MEDLINE, Embase, The Cochrane Library, CINAHL, Scopus, SportDISCUS, Web of Science Core Collection and PEDro from inception until November 2020. Two registries (ClinicalTrials.gov and REBEC) will also be searched for potential results in unpublished studies. There will be no restriction on language, date of publication, publication status or sample size. We will include randomised controlled trials enrolling adult HTx recipients with the presence of at least one exercise training group, which might be compared with another training modality and/or a non-exercise control group for a minimum of 4 weeks of intervention. The primary outcomes will be peak oxygen consumption and occurrence of adverse events. As secondary outcomes, the interaction between pulmonary ventilation, pulmonary perfusion and cardiac output, oxygen uptake efficiency slope, heart rate response, oxygen pulse, peak blood pressure and peak subjective perception of effort. In addition, we will evaluate the 6 min walking distance, health-related quality of life, endothelial function, muscle strength, body fat percentage and lean mass. Risk of bias will be assessed using the Cochrane RoB V.2.0 tool, and we plan to use the Confidence in Network Meta-Analysis tool to assess confidence in the results. All materials (raw data, processed data, statistical code and outputs) will be shared in a public repository.

Ethics and dissemination Given the nature of this study, no ethical approval will be required. We believe that the findings of this study may show which is the most efficacious and safe physical training modality for HTX

\section{Strengths and limitations of this study}

- This protocol was guided by Preferred Reporting Items for Systematic Review and Meta-Analysis Protocols statement, registered in the PROSPERO database, and Open Science Framework platform.

- We will perform a comprehensive literature review with no restrictions on language, publication date, publication status or sample size.

- The study will be guided by the Cochrane Handbook for Systematic Reviews of Interventions, V.6.1, in order to enhance the quality of the study.

- A potential limitation, inherent in the methodology of this study, is that indirect comparisons provide observational evidence across randomised trials and may suffer from the potential biases of observational studies such as confounding bias.

recipients. The completed systematic review and network meta-analysis will be submitted to a peer-reviewed journal.

PROSPERO registration number CRD42020191192.

\section{INTRODUCTION}

Heart transplantation (HTx) is the treatment of choice for selected patients with end-stage heart failure, representing the pinnacle of available therapy. ${ }^{1}$ Although transplantation improves quality of life and increases life expectancy, ${ }^{23}$ HTx recipients frequently experience impaired functional capacity, ${ }^{4}$ in addition to other complications inherent to the use of immunosuppressants. ${ }^{5}$ Reduced exercise tolerance, measured by peak oxygen consumption (peak $\mathrm{VO}_{2}$ ), occurs secondary to damage to both the central (cardiac and pulmonary) and peripheral (vascular and skeletal muscle) components. ${ }^{6}$

In this sense, concomitantly with prevention of HTx-related complications and 
control of cardiac risk factors, a structured exercise-based rehabilitation programme is recommended and may be an adequate strategy to assist in secondary prevention in these patients. ${ }^{7-9}$ Evidence suggests a multifactorial beneficial effect of exercise training (ET) in HTx recipients. ${ }^{6}$ Small randomised controlled trials have shown that rehabilitation improves autonomic control (both cardiac and peripheral),${ }^{10}$ muscle strength and body composition, ${ }^{11}$ while for endothelial function, synthesis of a small body of evidence shows high heterogeneity and the effect remains unclear. ${ }^{12}$ Additionally, in a Cochrane systematic review and meta-analysis, ET was efficacious for increasing peak $\mathrm{VO}_{2} \cdot{ }^{13}$ However, considering the broader spectrum of exercise interventions, it is unknown whether any modality is superior in terms of efficacy or potential for harm. In addition, synthesising the effect of ET on other clinically relevant outcomes will assist in understanding the therapeutic potential of ET in secondary prevention in this population.

Different ET modalities have been studied in patients after HTx, such as endurance training (moderate-intensity continuous and high-intensity interval training), ${ }^{13-15}$ resistance training ${ }^{11}{ }^{16}$ and the combination of both. ${ }^{17} 18$ Indeed, combined ET is the most recommended modality for cardiovascular rehabilitation, despite the lack of robust evidence of its superiority over other modalities in this specific population. ${ }^{89}$ In addition, there is little information about the characteristics of ET (eg, frequency, intensity, volume and type) as well as differences in adaptation depending on whether training is begun early or late after surgery. Even less is known about the true effect of ET on other relevant parameters beyond peak $\mathrm{VO}_{2}$ in the HTx scenario.

In patients with heart failure, some variables measured by cardiopulmonary exercise testing (CPET), such as peak $\mathrm{VO}_{2}$, the ratio of minute ventilation (VE) to carbon dioxide production $\left(\mathrm{VE} / \mathrm{VCO}_{2} \text { slope }\right)^{19}{ }^{20}$ and heart rate (HR) recovery after $\mathrm{ET}^{21}$ were identified as important prognostic markers. However, in HTx, the evidence base is much less clear. In a retrospective study, peak $\mathrm{VO}_{2}$ and self-reported functional capacity were found to be strong predictors of survival in HTx recipients. ${ }^{22}$ In turn, muscle strength and body fat seem to influence exercise capacity. ${ }^{23}$ Interestingly, while the main limiting symptom for ET before HTx was dyspnoea, after the procedure, patients reported interruption of exercise due to leg fatigue and muscle exhaustion. ${ }^{4}$ In addition, another important factor is the chronotropic response to $\mathrm{ET}^{24}$ due to the involvement of a denervated heart in increasing HR, contributing to the reduction of exercise tolerance and influencing adaptations to ET. ${ }^{25} 26$

In this systematic review and network meta-analysis, we will compare the safety and efficacy of different modalities of ET based on peak $\mathrm{VO}_{2}$ improvement. Furthermore, we will quantify the effect of ET on important outcomes that have not yet been scrutinised, while exploring factors that may influence physiological adaptations to ET.
Objectives

\section{Primary objectives}

- To compare the efficacy of different ET modalities (moderate-intensity continuous training, highintensity interval training, resistance training and combined aerobic plus resistance training considering both centre-based and home-based ET) in improving peak $\mathrm{VO}_{2}$ in $\mathrm{HTx}$ recipients;

- To compare rates of adverse events, such as vertigo, dizziness, musculoskeletal complaints, syncope, hypotension, elevated blood pressure or cardiovascular events (angina, arrhythmias, myocardial infarction, stroke and death), during and after sessions of different ET modalities.

\section{Secondary objectives}

- To compare quantitatively, through meta-analysis (ET vs usual care) and, if possible, through network metaanalysis, the efficacy of moderate-intensity continuous training, high-intensity interval training, resistance training and combined training (centre-based and home-based ET) in regards to the following variables: $\mathrm{VE} / \mathrm{VCO}_{2}$ slope, oxygen uptake efficiency slope (OUES), HR (rest, peak and recovery), peak oxygen pulse $\left(\mathrm{VO}_{2} / \mathrm{HR}\right)$, peak systolic and diastolic blood pressure, Borg Rating of Perceived Exertion scale, 6 min walk test distance, health-related quality of life, endothelial function, muscle strength and fat and lean mass percentage.

- To compare quantitatively, through meta-analysis (ET vs usual care), the efficacy and safety of ET in the following subgroups: patients after recent $(<6$ months) versus late HTx and those receiving centrebased versus home-based ET as well as at different follow-up periods.

\section{METHODS}

This protocol was guided by the Preferred Reporting Items for Systematic Review and Meta-Analysis Protocols (PRISMA-P) 2015 statement $^{27}$ (see checklist in online supplemental material 1) and the PRISMA-P 2015 Explanation and Elaboration Document. ${ }^{28}$ The same documents, as well as the PRISMA Extension for Network Meta-Analysis of Health Care Interventions, ${ }^{29}$ will be used to prepare the final report. In addition, the study will be conducted according to the Cochrane Handbook for Systematic Reviews of Interventions V.6.1 ${ }^{30}$ This systematic review and network meta-analysis were registered in the International Prospective Register of Systematic Reviews. All study materials will be shared publicly through the Open Science Framework tool, available at: https://osf.io/3rwxb/.

\section{Eligibility criteria}

This systematic review will be based on population, intervention, comparator, outcome and setting criteria.

\section{Participants}

(a) HTx recipients aged $\geq 18$ years, regardless of sex and race, (b) patients receiving immunosuppressive therapy 
according to the transplant centre protocol, who did not experience severe complications or high-grade rejection on cardiac biopsies during the ET period. Studies enroling heterotopic transplant recipients or multiorgan transplant recipients will be excluded.

\section{Interventions}

We will consider ET as a single strategy or as a component of a comprehensive cardiac rehabilitation programme (after hospital discharge), considering a minimum intervention period of 4 weeks. Centre-based and home-based interventions will also be considered for the following modalities: moderate-intensity continuous training, high-intensity interval training, resistance training and combined training (aerobic plus resistance).

\section{Comparators}

For network meta-analysis, by the very nature of this study, we will compare the eligible interventions among themselves. For the parallel meta-analysis, we will compare the interventions with their respective control groups (non-ET or usual care).

\section{Outcomes}

\section{Primary outcomes}

Peak $\mathrm{VO}_{2}$ measured through CPET in $\mathrm{L} / \mathrm{min}$ and $\mathrm{mL} /$ $\mathrm{kg} / \mathrm{min}$, whenever available. Rate of adverse events through the absolute and relative frequency of occurrences described in the safety outcomes.

\section{Secondary outcomes}

Other CPET variables: $\mathrm{VO}_{2} / \mathrm{HR}$ in $\mathrm{mL} /$ beat and both slopes, $\mathrm{VE} / \mathrm{VCO}_{2}$ slope and OUES as absolute measures. HR (rest, peak and recovery) in beats/min, peak systolic and diastolic blood pressure in $\mathrm{mm} \mathrm{Hg}$ and Borg Rating of Perceived Exertion score. Other secondary outcomes: 6 min walk distance (in metres), health-related quality of life using validated instruments (eg, the 36-Item ShortForm Health Survey and WHO Quality of Life questionnaire) and endothelial function by absolute $(\mathrm{mm})$ and relative (\%) flow-mediated dilation. Upper and lower extremity maximal strength in kilograms assessed using one-repetition maximum (1RM) testing or another equivalent method (eg, isokinetic evaluation $(\mathrm{Nm})$, sit-to-stand movements in $1 \mathrm{~min}$ and hand grip strength test). Relative $(\%)$ and absolute $(\mathrm{kg})$ fat mass and lean mass, preferably measured through dual-energy X-ray absorptiometry or bioelectrical impedance analysis.

\section{Safety outcomes}

Whenever data are available in the randomised controlled trial, we will quantitatively analyse the occurrence of adverse events-such as vertigo, dizziness, musculoskeletal complaints, syncope, hypotension, elevated blood pressure or cardiovascular events (angina, arrhythmias, myocardial infarction, stroke and death)—during and after exercise sessions.

\section{Study designs}

Only randomised controlled trials (parallel-group, crossover or cluster design) will be included. Crossover trials will be considered in their full form only if there is a washout period of at least 4 weeks. No restrictions will be imposed on language or date of publication.

\section{Information sources and search \\ Electronic search strategies}

For a comprehensive survey of the literature, the following databases from inception to November 2020 will be searched: PubMed/MEDLINE, Cochrane Library, Embase, the Cumulative Index to Nursing and Allied Health Literature (CINAHL), Scopus, SportDISCUS, Web of Science Core Collection and Physiotherapy Evidence Database (PEDro). Two registries (ClinicalTrials.gov and REBEC) will also be searched for potential results in unpublished studies. We will also review the grey literature, which includes repositories of dissertations and theses, conference publications and preprint repositories and databases. Authors will be contacted if further data are required. A hand search of the reference lists of included studies will be also conducted.

\section{Search strategy}

The main electronic search strategy was designed for PubMed/MEDLINE and will be adapted as appropriate for each of the other databases. Queries will be developed using Medical Subject Headings (MeSH) terms and their synonyms and Boolean operators (where possible) to improve searches. Keywords and MeSH terms include: 'heart transplantation', 'exercise', 'resistance training', 'physical endurance' and 'circuit-based exercise'. Comprehensive search strategies for all the databases that will be consulted are included in the online supplemental material 2.

\section{Study records}

Data managements and selection process

Data extraction will be based on the following steps: (1) In Clarivate Analytics Endnote X9 (2018) reference management software, the reviewer $(\mathrm{FF})$ will set up a library to gather all studies retrieved from the aforementioned databases, (2) all duplicates will be excluded, (3) the titles and abstracts will be evaluated by two independent reviewers (FF and JBdL) for classification as potentially eligible or non-eligible. Divergences will be solved by consensus between reviewers, and, if necessary, a third opinion (RS) will be requested, (4) studies classified as potentially eligible will be read in full and discrepancies will be solved by the same previous method, (5) the studies excluded in the previous stage will be compiled in an Excel worksheet, followed by their respective reasons for exclusion (no design of interest, no population of interest, no intervention of interest, no endpoints of interest or other). The results of the selection process will be presented in a flow diagram, as shown in the online supplemental material 3 . 


\section{Data collection process and data items}

Data will be extracted by two independent reviewers (FF and JBdL). Disagreements will be solved by consensus, and, if necessary, a third opinion (RS) will be requested. The reviewers will not be blinded to the authors' names, institutions or periodicals. The following information will be extracted:

- Study characteristics: first author, journal's name, year of publication, conflict of interest, publication type, study design (parallel, crossover or cluster randomised controlled trial), washout period (weeks), study period (weeks), country, language of the publication and number of patients randomised;

- Patient baseline characteristics: age, weight, height, body mass index, sex, time since transplantation, immunosuppressant therapy, comorbidities, aetiology and duration of heart failure, surgical technique, previous exercise-based rehabilitation (phase 1) and presence of possible additional interventions to training; in addition, outcome assessment methods; equipment used (cycle ergometer or treadmill) and peak respiratory exchange ratio, when CPET is performed;

- Interventions and comparators: training modality, material resources, intended target zone and form of intensity control, session volume, weekly frequency and follow-up period; supervisory level information, if centre-based or home-based ET.

- Results: number of participants in each group, preintervention and postintervention values, deltas, standard deviations or other measures of dispersion and $p$ values.

\section{Geometry of the network}

The forest.netmeta function of the netmeta package will be used to build and present the geometry of different interventions. In the graph, nodes will be used to represent the intervention and edges to show comparisons between interventions. Besides qualitative descriptions and graphs, we will provide quantitative metrics assessing features of network geometry, such as diversity, co-occurrence and homophily.

\section{Risk of bias within individual studies}

The risk of bias will be assessed using the Cochrane RoB V.2.0 tool. ${ }^{31}$ The assessment of the studies will be performed independently by two reviewers (DdSS and SATL); any disagreements will be resolved by consensus or by discussion with a third researcher (JBdL). Evaluation of quality will be divided into five items: (1) bias arising from the randomisation process, (2) bias due to deviations from intended interventions, (3) bias due to missing outcome data, (4) bias in measurement of the outcome, (5) bias in selection of the reported result. Also, the risk of bias will be classified into three categories: (1) low risk of bias, (2) some concerns and (3) high risk of bias.

\section{Publication bias}

To investigate the influence of small-study effects, we will use the visual inspection method of funnel plot if at least 10 studies are included in a meta-analysis, followed by Egger's test. ${ }^{32}$

\section{Data synthesis}

Main analyses

We will use difference in means as the principal summary measure of the effect to express comparisons between interventions, associated with the $95 \%$ confidence interval as a measure of uncertainty. When the same outcome is presented by different measures, the standardised mean difference will be applied. As a summary measure of dichotomous variables, we will use the risk ratio. If quantitative synthesis is not appropriate, a systematic narrative synthesis will be provided.

For continuous variables, we will extract the mean (or other measure of central tendency) and standard deviation (or other measure of dispersion) of variables at baseline and in the follow-up in each arm of the studies. When available, we plan to obtain the mean change from baseline and SD or other measures of dispersion in each arm of the trials. For dichotomous outcomes, we will collect absolute and relative frequencies in each treatment arm.

\section{Planned methods of analysis}

We plan to use the netmeta package V.1.2-1 implemented in R V.3.6.2 software for Mac to perform a network metaanalysis $^{33}$ and synthesise direct and indirect evidence of the therapeutic effects of the interventions. Nodesplitting method will be used to assess inconsistencies between direct and indirect comparisons when observing a loop connecting three arms. We will present a treatment ranking by $\mathrm{p}$ scores based on the point estimates and standard error of the available network.

\section{Additional analyses}

We plan to perform analyses to compare quantitatively, through meta-analysis (ET vs usual care), the efficacy and safety of ET in two subgroups: patients after recent (less than 6 months) versus late HTx and centre-based versus home-based ET as well as different follow-up periods. In case of significant heterogeneity or inconsistency, a subgroup analysis will be performed to explore, when possible, the following variables: age, sex and comorbidities. Finally, a sensitivity analysis will be performed for the inclusion of studies with high risk of bias and/or missing data.

\section{Risk of bias across studies}

We plan to use the Confidence in Network Meta-Analysis (CINeMA) tool ${ }^{34} 35$ to assess confidence in the results. CINeMA considers six domains-within-study bias, reporting bias, indirectness, imprecision, heterogeneity and incoherence-and assigns judgements at three levels (no concerns, some concerns or major concerns). For each treatment effect, adjudicate levels of confidence corresponding to the usual Grading of Recommendations, 
Assessment, Development, and Evaluation assessments of very low, low, moderate, or high will be used.

\section{Patient and public involvement}

No patient involved.

\section{Ethics and dissemination}

Given the nature of this study, no ethical approval will be required. The completed systematic review and network meta-analysis will be submitted to a peer-reviewed journal.

\section{Author affiliations}

${ }^{1}$ Graduate Program in Cardiology and Cardiovascular Sciences, Universidade Federal do Rio Grande do Sul, Hospital das Clínicas de Porto Alegre, Porto Alegre, RS, Brazil

${ }^{2}$ Exercise Cardiology Research Group, Universidade Federal do Rio Grande do Sul, Hospital de Clínicas de Porto Alegre, Porto Alegre, RS, Brazil

${ }^{3}$ Interdisciplinary Research Group in Translational Cardiology, Clinical Research Center, Hospital das Clínicas de Porto Alegre, Porto Alegre, RS, Brazil

${ }^{4}$ Department of Evidence-Based Health, Brazilian Cochrane Center, Universidade Federal de São Paulo, São Paulo, SP, Brazil

${ }^{5}$ Heart Failure and Cardiac Transplant Unit, Cardiology Division, Hospital das Clínicas de Porto Alegre, Porto Alegre, Rio Grande do Sul, Brazil

${ }^{6}$ Associate Professor, School of Medicine, Universidade Federal do Rio Grande do Sul, Porto Alegre, RS, Brazil

Correction notice This article has been corrected since it first published. The provenance and peer review statement has been included.

Twitter Juliana Beust de Lima @beust_lima

Acknowledgements The authors of this protocol thank Bárbara Pilatti Piffer, staff librarian at Universidade Federal do Rio Grande do Sul, for her contributions in design of the search strategies and retrieval of documents.

Contributors Conception of the study: JBdL, DdSS, FF, SATL, RS. Major drafters of the protocol: JBdL, DdSS, FF, NCJ, RS. Minor drafters of the protocol: SATL, GC provided feedback to the protocol: LAG, NC, RS. Data extraction and synthesis: FF, NCJ, JBdL.

Funding This protocol was partially supported by the Hospital de Clínicas de Porto Alegre Research Incentive Fund (FIPE-HCPA), Porto Alegre, Brazil-project number 2018-0292. JBdL and GC are granted by the Conselho Nacional de Pesquisa e Desenvolvimento (CNPq). DdSS and FF are granted by the Coordenação de Aperfeiçoamento de Pessoal de Nivel Superior (CAPES). This study is conducted by an academic institution and a research group that has no relationship with the pharmaceutical industry.

Competing interests None declared.

Patient consent for publication Not required.

Provenance and peer review Not commissioned; externally peer reviewed.

Supplemental material This content has been supplied by the author(s). It has not been vetted by BMJ Publishing Group Limited (BMJ) and may not have been peer-reviewed. Any opinions or recommendations discussed are solely those of the author(s) and are not endorsed by BMJ. BMJ disclaims all liability and responsibility arising from any reliance placed on the content. Where the content includes any translated material, BMJ does not warrant the accuracy and reliability of the translations (including but not limited to local regulations, clinical guidelines, terminology, drug names and drug dosages), and is not responsible for any error and/or omissions arising from translation and adaptation or otherwise.

Open access This is an open access article distributed in accordance with the Creative Commons Attribution Non Commercial (CC BY-NC 4.0) license, which permits others to distribute, remix, adapt, build upon this work non-commercially, and license their derivative works on different terms, provided the original work is properly cited, appropriate credit is given, any changes made indicated, and the use is non-commercial. See: http://creativecommons.org/licenses/by-nc/4.0/.

Author note Statement: The protocol of this network meta-analysis was guided by the Preferred Reporting Items for Systematic Review and Meta-Analysis Protocols (PRISMA-P) 2015 statement and the PRISMA-P 2015 Explanation and Elaboration Document. Amendments: This is the first version of the protocol. Any amendments to the protocol will be updated and published on the PROSPERO database (CRD42020191192).

\section{ORCID iDs}

Juliana Beust de Lima http://orcid.org/0000-0002-5408-2457

Douglas dos Santos Soares http://orcid.org/0000-0002-9166-7614

Filipe Ferrari http://orcid.org/0000-0001-6929-8392

Nelson Carvas Junior http://orcid.org/0000-0003-2168-8927

Gabriel Carvalho http://orcid.org/0000-0001-7792-826X

Santiago Alonso Tobar Leitão http://orcid.org/0000-0002-4163-7783

Lívia Adams Goldraich http://orcid.org/0000-0002-1523-4286

Nadine Clausell http://orcid.org//0000-0003-4207-3809

Ricardo Stein http://orcid.org/0000-0003-2357-5176

\section{REFERENCES}

1 Yancy CW, Jessup M, Bozkurt B, et al. 2013 ACCF/AHA guideline for the management of heart failure: a report of the American College of cardiology Foundation/American heart association Task force on practice guidelines. J Am Coll Cardiol 2013;62:e147-239.

2 Grady KL, Jalowiec A, White-Williams C. Improvement in quality of life in patients with heart failure who undergo transplantation. $J$ Heart Lung Transplant 1996;15:749-57.

3 Khush KK, Cherikh WS, Chambers DC, et al. The International Thoracic Organ Transplant Registry of the International Society for Heart and Lung Transplantation: Thirty-sixth adult heart transplantation report - 2019; focus theme: Donor and recipient size match. J Heart Lung Transplant 2019;38:1056-66. Epub 2019 Aug 10. Erratum in: $J$ Heart Lung Transplant. 2020;39(1):91.

4 Habedank D, Ewert R, Hummel M, et al. Changes in exercise capacity, ventilation, and body weight following heart transplantation. Eur J Heart Fail 2007;9:310-6.

$5 \mathrm{Kim} \mathrm{IC,} \mathrm{Youn} \mathrm{JC,} \mathrm{Kobashigawa} \mathrm{JA.} \mathrm{The} \mathrm{past,} \mathrm{present} \mathrm{and} \mathrm{future} \mathrm{of}$ heart transplantation. Korean Circ J 2018;48:565-90.

6 Tucker WJ, Beaudry RI, Samuel TJ, et al. Performance limitations in heart transplant recipients. Exerc Sport Sci Rev 2018;46:144-51.

7 Mezzani A, Hamm LF, Jones AM, et al. Aerobic exercise intensity assessment and prescription in cardiac rehabilitation: a joint position statement of the European association for cardiovascular prevention and rehabilitation, the American association of cardiovascular and pulmonary rehabilitation and the Canadian association of cardiac rehabilitation. Eur J Prev Cardiol 2013;20:442-67.

8 Herdy AH, López-Jiménez F, Terzic CP, et al. South American guidelines for cardiovascular disease prevention and rehabilitation. Arq Bras Cardiol 2014;103:1-31.

9 Ambrosetti M, Abreu A, Corrà U, et al. Secondary prevention through comprehensive cardiovascular rehabilitation: from knowledge to implementation. 2020 update. A position paper from the secondary prevention and rehabilitation section of the European association of preventive cardiology. Eur J Prev Cardiol 2020;30:204748732091337.

10 Bernardi L, Radaelli A, Passino C, et al. Effects of physical training on cardiovascular control after heart transplantation. Int $\mathrm{J}$ Cardiol 2007;118:356-62.

11 Braith RW, Welsch MA, Mills RM, et al. Resistance exercise prevents glucocorticoid-induced myopathy in heart transplant recipients. Med Sci Sports Exerc 1998;30:483-9.

12 de Souza JAF, Araújo BTS, de Lima GHC, et al. Effect of exercise on endothelial function in heart transplant recipients: systematic review and meta-analysis. Heart Fail Rev 2020;25:487-94.

13 Anderson L, Nguyen TT, Dall CH, et al. Exercise-based cardiac rehabilitation in heart transplant recipients. Cochrane Database Syst Rev 2017;4:CD012264.

14 Perrier-Melo RJ, Figueira FAMDS, Guimarães GV, et al. High-Intensity interval training in heart transplant recipients: a systematic review with meta-analysis. Arq Bras Cardiol 2018;110:188-94.

15 Nytrøen K, Rolid K, Andreassen AK. Effect of High-Intensity Interval Training in De Novo Heart Transplant Recipients in Scandinavia. Circulation. 2019 May 7;139(19):2198-2211. Erratum in: Circulation. 2019 Oct 22;140(17):e737, 2019

16 Braith RW, Mills RM, Welsch MA, et al. Resistance exercise training restores bone mineral density in heart transplant recipients. J Am Coll Cardiol 1996;28:1471-7.

17 Haykowsky M, Taylor D, Kim D, et al. Exercise training improves aerobic capacity and skeletal muscle function in heart transplant recipients. Am J Transplant 2009;9:734-9.

18 Kobashigawa JA, Leaf DA, Lee N. A controlled trial of exercise rehabilitation after heart transplantation [published correction 
appears in N Engl J Med 1999 Mar 25;340(12):976]. N Engl J Med 1999;340:272-7.

19 Arena R, Myers J, Aslam SS, et al. Peak VO2 and VE/VCO2 slope in patients with heart failure: a prognostic comparison. Am Heart $J$ 2004;147:354-60.

20 Cahalin LP, Chase P, Arena R, et al. A meta-analysis of the prognostic significance of cardiopulmonary exercise testing in patients with heart failure. Heart Fail Rev 2013;18:79-94.

21 Arena R, Guazzi M, Myers J, et al. Prognostic value of heart rate recovery in patients with heart failure. Am Heart $J$ 2006;151:851. e7-851.e13.

22 Yardley M, Havik OE, Grov I, et al. Peak oxygen uptake and selfreported physical health are strong predictors of long-term survival after heart transplantation. Clin Transplant 2016;30:161-9.

23 Nytrøen K, Rustad LA, Gude E, et al. Muscular exercise capacity and body fat predict VO(2peak) in heart transplant recipients. Eur J Prev Cardiol 2014;21:21-9.

24 Gullestad L, Haywood G, Ross H, et al. Exercise capacity of heart transplant recipients: the importance of chronotropic incompetence. $J$ Heart Lung Transplant 1996;15:1075-83.

25 Carvalho VO, Bocchi EA, Pascoalino LN, et al. The relationship between heart rate and oxygen consumption in heart transplant recipients during a cardiopulmonary exercise test: heart rate dynamic during exercise test. Int J Cardiol 2010;145:158-60.

26 Ciolac EG, Castro RE, Marçal IR, et al. Cardiac reinnervation affects cardiorespiratory adaptations to exercise training in individuals with heart transplantation. Eur J Prev Cardiol 2020;27:1151-61.
27 Moher D, Shamseer L, Clarke M, et al. Preferred reporting items for systematic review and meta-analysis protocols (PRISMA-P) 2015 statement. Syst Rev 2015;4:1.

28 Liberati A, Altman DG, Tetzlaff J, et al. The PRISMA statement for reporting systematic reviews and meta-analyses of studies that evaluate health care interventions: explanation and elaboration. PLOS Med 2009;6:e1000100.

29 Hutton B, Salanti G, Caldwell DM, et al. The PRISMA extension statement for reporting of systematic reviews incorporating network meta-analyses of health care interventions: checklist and explanations. Ann Intern Med 2015;162:777-84.

30 et alHiggins JPT, Thomas J, Chandler J. Cochrane Handbook for Systematic Reviews of Interventions version 6.1 (updated September 2020). Cochrane, 2020. Available: www.training.cochrane.org/ handbook

31 Sterne JAC, Savović J, Page MJ, et al. Rob 2: a revised tool for assessing risk of bias in randomised trials. BMJ 2019;366:14898.

32 Egger M, Davey Smith G, Schneider M, et al. Bias in meta-analysis detected by a simple, graphical test. BMJ 1997;315:629-34.

33 Schwarzer G, Carpenter JR, Rücker G. Meta-Analysis with R, use R! Switzerland: Springer, 2015: p. 187-216.

34 Nikolakopoulou A, Higgins JPT, Papakonstantinou T, et al. Cinema: an approach for assessing confidence in the results of a network meta-analysis. PLoS Med 2020;17:e1003082.

35 Papakonstantinou T, Nikolakopoulou A, Higgins JPT, et al. Cinema: software for semiautomated assessment of the confidence in the results of network meta-analysis. Campbell Syst Rev 2020;16. 